\title{
COVID-19 in Liver Transplant Patients: Report of 2 Cases and Review of the Literature
}

This article was published in the following Dove Press journal:

International Medical Case Reports Journal

\author{
Ramin Niknam (ID) \\ Seyed Ali Malek-Hosseini ${ }^{2}$ \\ Seyed Saeid Hashemieh' \\ Masoud Dehghani ${ }^{2}$ \\ 'Gastroenterohepatology Research \\ Center, Shiraz University of Medical \\ Sciences, Shiraz, Iran; ${ }^{2}$ Shiraz Transplant \\ Center, Abu Ali Sina Hospital, Shiraz \\ University of Medical Sciences, Shiraz, \\ Iran
}

\begin{abstract}
Coronavirus 2019 (COVID-19) is caused by a novel coronavirus. Although liver injury is common in patients with COVID-19, little is known about its clinical presentation and management in liver transplantation. This is the first report from Iran that presented two cases of liver transplantation with COVID-19, neither of which had pulmonary signs and symptoms, but after diagnostic imaging, both had lung involvement. We also reviewed some literature on the management of COVID-19 in liver transplant patients. In conclusion, our patients improved after starting hydroxychloroquine and continuing to take all immunosuppressive agents except mycophenolate based on the multidisciplinary team's decision.
\end{abstract}

Keywords: COVID-19, coronavirus, liver transplantation, immunosuppressant, management

\section{Introduction}

Coronavirus 2019 (COVID-19) is caused by a novel coronavirus. ${ }^{1,2}$ Clinical presentation and management of liver injury in patients with COVID-19 in liver transplantation is unclear and may be an increasing challenge for the transplant centers. $^{3,4}$

In this report, we have described 2 liver transplant cases with COVID-19. We also reviewed some literature on the management of COVID-19 in liver transplant patients.

\section{Case I}

A 60-year-old woman with diabetes mellitus has been referred to the transplant center at Abu Ali Sina hospital, the main center of liver transplantation in Iran, due to intermittent fever, weakness, and anorexia. She underwent liver transplant 10 months before her recent visit due to autoimmune hepatitis. All symptoms started 2 weeks before being referred to our hospital. The patient had no history of any respiratory symptoms include cough and dyspnea. There was no history of high blood pressure or coronary artery disease. She was taking medications including mycophenolate ( $720 \mathrm{mg}$ per day), tacrolimus ( $2 \mathrm{mg}$ per day), prednisolone (15 $\mathrm{mg}$ per day), folic acid (1 $\mathrm{mg}$ per day), and ursodeoxycholic acid (900 mg per day). The patient's vital signs were oral temperature, $37.9^{\circ} \mathrm{C}$, heart rate of 105 beats per minute, respiration rate of 16 beats per minute, blood pressure of 110/70 $\mathrm{mm} \mathrm{Hg}$. On physical examination, the patient had jaundice, but lung and other organs were normal. The patient admitted to hospital for more evaluation and samples of nasal and pharyngeal swabs for COVID-19 diagnosis were tested using realtime polymerase chain reaction (RT-PCR), which showed positive results. Computed tomography (CT) scan of the chest was performed, in which multiple bilateral nodular
Correspondence: Masoud Dehghan Shiraz Transplant Center, Abu Ali Sina Hospital, Shiraz University of Medical

Sciences, Shiraz, Iran

Tel +989131415796

Email dehghanimasood@yahoo.com
International Medical Case Reports Journal 2020:13 3|7-32| 
infiltrates of varying sizes and a few patchy ground-glass opacity were observed (Figure 1). Table 1 shows the patient's laboratory results. After discussing the patient's condition in a multidisciplinary team, the patient was transferred to isolated room, mycophenolate was discontinued, and meropenem (1g IV q.8h), co-trimoxazole (400/80) one tablet twice daily, and hydroxychloroquine (HCQ) (400 mg/day) were started for 14 days. According to the cholestatic pattern of liver tests, ultrasound and magnetic resonance cholangiopancreatography were performed, the results of which were in favor of common bile duct stricture, and radiological intervention was considered to treat this problem. During the hospitalization period, the patient did not show any pulmonary symptoms. Although the patient's condition improved after 5 weeks of follow-up, due to the concomitant treatment of bile duct stenosis and COVID-19 in this patient, it is difficult to separate the effect and contribution of treatment of the two diseases in the patient's recovery.

\section{Case 2}

A 46-year-old man was admitted to the post-transplant ward at Abu Ali Sina hospital due to fever, severe headache, anorexia, nausea, and vomiting. He underwent liver transplant 2 years ago due to budd chiari syndrome. All symptoms started 7 days before admission. The patient had no history of any respiratory symptoms. There was no history of diabetes, high blood

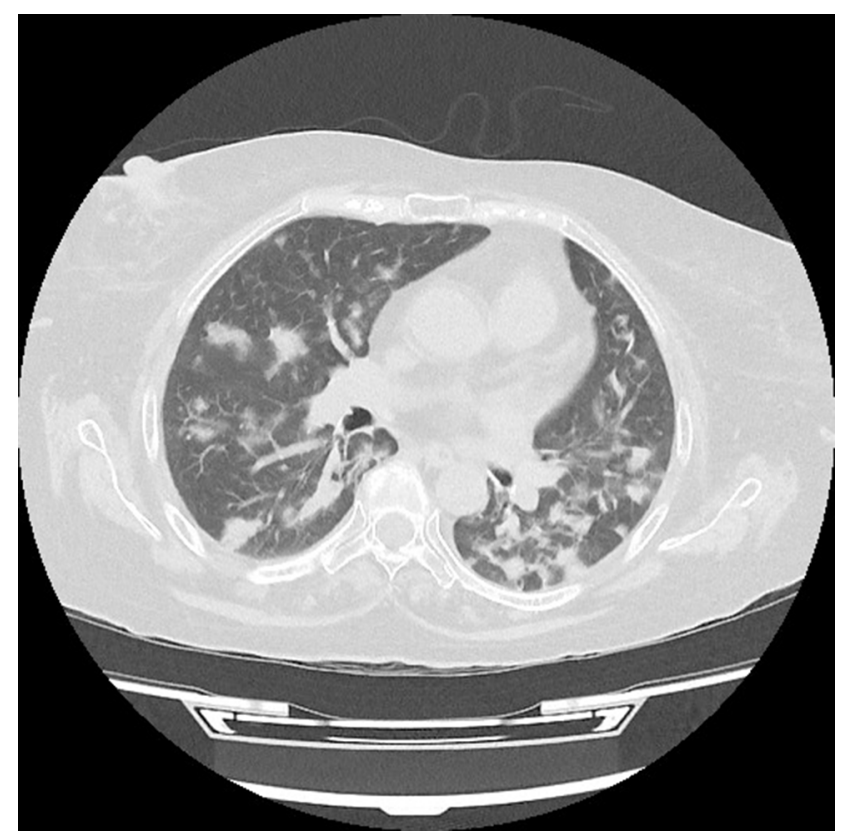

Figure I Computed tomography scan of the chest of a liver transplant woman with coronavirus 2019 (COVID-19) showed multiple bilateral nodular infiltrates of varying sizes and a few patchy ground-glass opacity.
Table I The Results of Laboratory Parameters of the Liver Transplant Patients with Coronavirus 2019 (COVID-19)

\begin{tabular}{|l|l|l|l|}
\hline Laboratory Data & $\begin{array}{l}\text { Case } \\
\text { I }\end{array}$ & Case 2 & Normal Range \\
\hline White blood cells $\left(/ \mathrm{mm}^{3}\right)$ & 6700 & 5.1 & $4000-10,000$ \\
Hemoglobin (g/dl) & 13.3 & $\mathrm{II} .1$ & $12-16$ \\
Platelets $\left(\mathrm{mm}^{3}\right.$ ) & 74,000 & 137,000 & $140,000-400,000$ \\
Direct bilirubin (mg/dl) & 18.8 & 0.45 & $<0.2$ \\
Total bilirubin (mg/dl) & 28.6 & 1.24 & $0.1-1.2$ \\
Aspartate aminotransferase(U/L) & 204 & 40 & $<31$ \\
Alanine aminotransferase (U/L) & 333 & 88 & $<31$ \\
Alkaline phosphatase (U/L) & 1140 & 244 & $64-306$ \\
Prothrombin Time. Patient (S) & 12 & 12 & 12.5 \\
Prothrombin Time. Control (S) 9 & 12 & 12 & 12.5 \\
Erythrocyte sedimentation rate & 48 & 61 & $<29$ \\
(mm/hr) & & & \\
\hline
\end{tabular}

pressure, or coronary artery disease. He was taking medications including mycophenolate (1440 mg per day), tacrolimus (3 $\mathrm{mg}$ per day), prednisolone (10 $\mathrm{mg}$ per day), folic acid (1 $\mathrm{mg}$ per day), and aspirin ( $80 \mathrm{mg}$ per day). The patient's vital signs on the day of admission were oral temperature, $38.7^{\circ} \mathrm{C}$, heart rate of 115 beats per minute, respiration rate of 15 beats per minute, blood pressure of 100/70 mm Hg. Physical examinations, including neurological and pulmonary, were normal. The brain magnetic resonance imaging was normal and the laboratory parameters are shown in Table 1 . The patient did not consent to the sampling through lumbar puncture. Due to unexplained fever in a patient with suppressed immunity and normal brain MRI, samples of nasal and pharyngeal swabs were taken for COVID-19 evaluation. Samples were tested using RT-PCR, which showed positive results. CT scan of the chest was performed, in which only a small peripheral patchy ground-glass opacity was observed in right lung (Figure 2). After discussing the patient's condition in a multidisciplinary team, the patient was transferred to an isolated room and received HCQ (400 mg/day) for 5 days. Mycophenolate was discontinued, while other patient's medications were continued. The patient's fever, headache, and nausea subsided 2 weeks later, and the patient's appetite improved by the fourth week.

\section{Discussion}

This is the first report from Iran that presented two cases of liver transplantation with COVID-19, neither of which had pulmonary symptoms, but after diagnostic imaging, both had lung involvement, one of which was severe.

The first cases of COVID-19 were identified in China. ${ }^{5}$ COVID-19 is caused by a novel virus called severe acute 


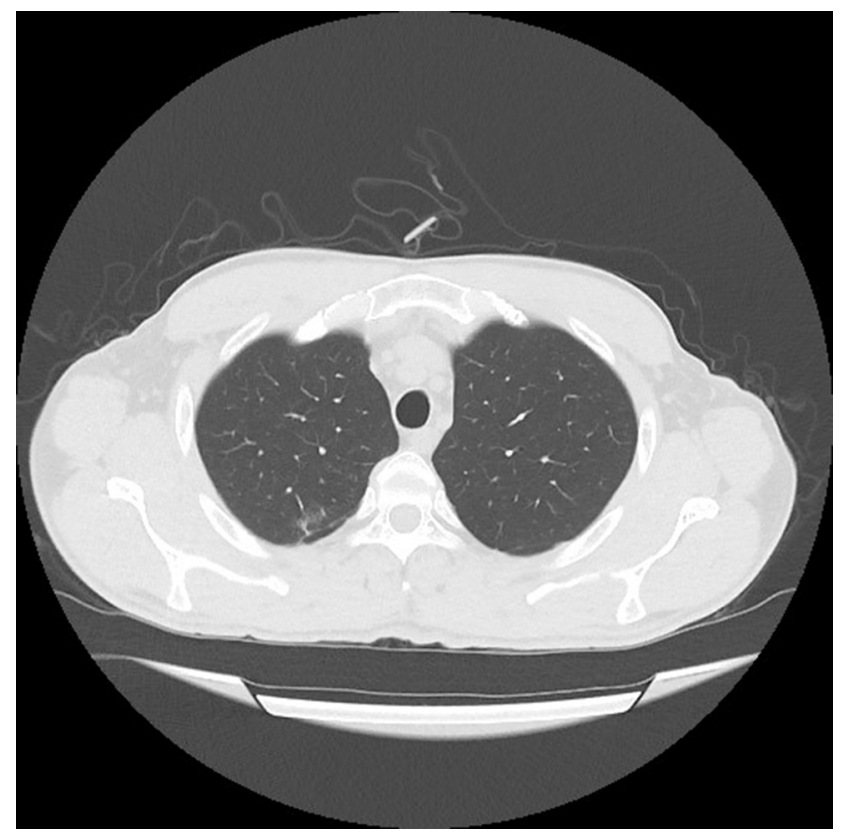

Figure 2 Computed tomography scan of the chest of a liver transplant man with coronavirus 2019 (COVID-19) showed a small peripheral patchy ground-glass opacity in right lung.

respiratory syndrome coronavirus 2 (SARS-CoV-2). This virus spread rapidly around the world and is now a public health emergency. ${ }^{2}$ The clinical features of COVID-19 patients are very diverse. Pulmonary symptoms are common in COVID-19, but some patients may show symptoms other than the respiratory tract, including neurological manifestations, and changes in smell and taste. ${ }^{1,6}$ One of our patients presented with severe headache as a sign of neurological disease.

Increased liver enzymes are a common problem in hospitalized patients with COVID-19. Liver injury is more common in severe cases of COVID-19 than in mild cases, which do not require special treatment in mild cases. It has been suggested that increasing liver enzymes in hospitalized patients should not be a contraindication to using the research or off-label therapies for COVID-19, but regular monitoring of liver enzymes should be performed. ${ }^{3}$ Although liver injury is common in patients with COVID-19, little is known about its clinical presentation and management in liver transplantation. ${ }^{3,4}$ In a report by Bhoori et al, 3 of the 111 longterm survivors of liver transplantation died in a few weeks following severe COVID-19. ${ }^{7}$ Huang et al described a case of COVID-19 in a liver transplant patient with symptoms of respiratory disease. Various therapies have been used for the patient, including nebulized $\alpha$-interferon, umifenovir, and lopinavir/ritonavir, methylprednisolone, antibiotic therapy, biliary drainage by endoscopic retrograde cholangiopancreatography, continuous renal replacement therapy, and plasma exchange. Their patient's condition progressed rapidly from mild to critical illness and was complicated by multi-organ failure, and he eventually died. ${ }^{8}$ Contrary to this report, none of our patients had respiratory symptoms.

In another report, Zhong et al described two cases of COVID-19 in liver transplant recipients that ultimately had good outcomes. Immunosuppression medications were discontinued for the first patient who had a liver rejection, but for the second case, immunosuppression agents were only reduced. ${ }^{9}$ Liu et al reported a case of COVID-19 in a liver transplant patient who presented with fever and multiple peripheral patchy ground-glass in CT scan. They tried various therapies for the patient, including umifenovir, lopinavir/ritonavir, methylprednisolone, intravenous immunoglobulin, antibiotic, and alpha interferon. Immunosuppression medications were also discontinued and eventually the patient recovered. ${ }^{10}$

Although there are concerns that organ transplant patients may be at greater risk for COVID-19 due to immunosuppressive therapy, there is still no conclusive evidence that the immunosuppressive protocol has been modified. ${ }^{11}$ Liver transplant recipients without COVID-19 may not need to adjust the dose of immunosuppressive medications. For patients with mild to moderate COVID-19, it is recommended that immunosuppression be continued, ${ }^{4}$ but if there is fever, lymphopenia, or worsening of the patient's pneumonia, dose reduction of azathioprine or mycophenolate should be considered. ${ }^{3}$ Patients with severe or rapidly progressing COVID-19 should be considered to reduce the dose of calcineurin inhibitor and discontinue antimetabolic medications. ${ }^{4}$ In our patients, mycophenolate was discontinued, while other immunosuppressive agents were continued. It is recommended that the high-dose prednisone be minimized, but at least a daily dose of $10 \mathrm{mg}$ prednisone or its equivalent should be used to prevent adrenal insufficiency. Corticosteroids or other immunosuppressive medications can be re-used with caution where the potential clinical benefit might be outweighed by the risks. ${ }^{3,4}$ In our report, low-dose prednisolone was continued for both patients. If patients need antipyretics, acetaminophen at a daily dose of $\leq 2 \mathrm{~g} /$ day is recommended. ${ }^{3}$ Although the use of HCQ and azithromycin is controversial for the treatment of liver transplant recipients with COVID- $19,{ }^{3}$ our multidisciplinary team decided to initiate HCQ for both patients. Although HCQ has been used in different diseases for many years, its mechanism of action is still on the rise. 
Some studies have reported that HCQ has antiviral activity against SARS-CoV-2, so due to the lack of a definite antiviral medication or vaccine, it was recommended as a potential intervention strategy for COVID-19 treatment. $^{3,12,13}$ However, HCQ has various side effects and warnings, including cardiac arrhythmia, heart failure, blindness, and renal toxicity, especially when taken in higher doses. ${ }^{12,14}$ Evidence currently does not support the use of lopinavir/ritonavir for the treatment of COVID-19 in liver transplant recipients, ${ }^{3,4}$ so we did not use this medication to treat our patients.

At a pediatric liver center in Latin America, patients were routinely screened for any clinical signs of COVID19 during the current outbreak. According to their published report, although patients theoretically had high-risk factors for COVID-19, including receiving multiple immunosuppressive mediations or autoimmune disease, only six patients developed mild upper airway infection and fever. None of the patients, including those with a positive SARS-CoV-2 test, developed clinical lung disease. They concluded that there are no reasons to delay life-saving treatments such as liver transplantation in children during the current coronavirus outbreak. ${ }^{15}$ It is recommended that all patients with severe COVID-19 be screened for hyperinflammation status using laboratory methods to identify the subgroup of participants for whom suppression of the immune system can improve mortality. ${ }^{16}$ It is not clear why, in mentioned reports, transplanted patients with COVID-19 have fewer complications than expected. One hypothesis is that SARS-CoV-2 may cause disease through the immune system, so the use of immunosuppressive mediations may reduce the complications by inhibiting the immune response. But further research is needed to prove this hypothesis.

In conclusion, none of our patients had respiratory symptoms, and lung imaging as well as RT-PCR tests were performed based on high clinical suspicion of COVID-19 for diagnosis. On the other hand, both patients improved after starting HCQ and continuing to take all immunosuppressive medications except mycophenolate. But data on clinical features and management of COVID-19 in the liver transplant patients are still very limited and more research is needed.

\section{Patient Consent}

Informed consent was obtained from the study participants prior to study commencement. They also consented for publication of their treatment and image details. This report does not contain personal information that could lead to the identification of patients. In addition, no institutional approval was required to publish the details of the cases. All organs were donated voluntarily with written informed consent, and that this was conducted in accordance with the Declaration of Istanbul.

\section{Disclosure}

The authors report no conflicts of interest in this work.

\section{References}

1. Guan WJ, Ni ZY, Hu Y, et al. Clinical characteristics of coronavirus disease 2019 in China. $N$ Engl J Med. 2020;382(18):1708-1720.

2. World Health Organization. Coronavirus disease situation reports. March 18, 2020. Available from: https://www.who.int/emergencies/ diseases/novel -coronavirus-2019/situation-reports. Accessed March 19, 2020.

3. Fix OK, Hameed B, Fontana RJ, et al. Clinical best practice advice for hepatology and liver transplant providers during the COVID-19 pandemic: AASLD expert panel consensus statement. Hepatology. 2020;72(1):287-304. doi:10.1002/hep.31281

4. Liu H, He X, Wang Y, et al. Management of COVID-19 in patients after liver transplantation: beijing working party for liver transplantation. Hepatol Int. 2020;14(4):432-436. doi:10.1007/ s12072-020-10043-z

5. Huang C, Wang Y, Li X, et al. Clinical features of patients infected with 2019 novel coronavirus in Wuhan, China. Lancet. 2020;395 (10223):497-506. doi:10.1016/S0140-6736(20)30183-5

6. Mao L, Jin H, Wang M, et al. Neurologic manifestations of hospitalized patients with coronavirus disease 2019 in Wuhan, China. JAMA Neurol. 2020;77(6):683. doi:10.1001/jamaneurol.2020.1127

7. Bhoori S, Rossi RE, Citterio D, Mazzaferro V. COVID-19 in long-term liver transplant patients: preliminary experience from an Italian transplant centre in Lombardy. Lancet Gastroenterol Hepatol. 2020;5(6):532-533. doi:10.1016/S2468-1253(20)30116-3

8. Huang JF, Zheng KI, George J, et al. Fatal outcome in a liver transplant recipient with COVID-19. Am J Transplant. 2020;20 (7):1907-1910. doi:10.1111/ajt.15909

9. Zhong Z, Zhang Q, Xia H, et al. Clinical characteristics and immunosuppressant management of coronavirus disease 2019 in solid organ transplant recipients. Am $J$ Transplant. 2020;20 (7):1916-1921. doi:10.1111/ajt.15928

10. Liu B, Wang Y, Zhao Y, Shi H, Zeng F, Chen Z. Successful treatment of severe COVID-19 pneumonia in a liver transplant recipient. $\mathrm{Am}$ J Transplant. 2020;20(7):1891-1895. doi:10.1111/ajt.15901

11. Saigal S, Gupta S, Sudhindran S, et al. Liver transplantation and COVID-19 (coronavirus) infection: guidelines of the liver transplant society of India (LTSI). Hepatol Int. 2020;14(4):429-431. doi:10.1007/s12072-020-10041-1

12. Alanagreh L, Alzoughool F, Atoum M. Risk of using hydroxychloroquine as a treatment of COVID-19. Int J Risk Saf Med. 2020;1-6. doi: $10.3233 /$ JRS-200024

13. Meo SA, Klonoff DC, Akram J. Efficacy of chloroquine and hydroxychloroquine in the treatment of COVID-19. Eur Rev Med Pharmacol Sci. 2020;24(8):4539-4547. doi:10.26355/eurrev_202 004_21038

14. Uzelac I, Iravanian S, Ashikaga H, et al. Fatal arrhythmias: another reason why doctors remain cautious about chloroquine/hydroxychloroquine for treating COVID-19. Heart Rhythm. 2020. doi:10.1016/j. hrthm.2020.05.030 
15. Tannuri U, Tannuri ACA, Cordon MNA, Miyatani HT. Low incidence of COVID-19 in children and adolescent post-liver transplant at a Latin American reference center. Clinics. 2020;75:e1986. doi:10.6061/clinics/2020/e1986
16. Mehta P, McAuley DF, Brown M, et al. COVID-19: consider cytokine storm syndromes and immunosuppression. Lancet. 2020;395 (10229):1033-1034. doi:10.1016/S0140-6736(20)30628-0

\section{Publish your work in this journal}

The International Medical Case Reports Journal is an international, peer-reviewed open-access journal publishing original case reports from all medical specialties. Previously unpublished medical posters are also accepted relating to any area of clinical or preclinica science. Submissions should not normally exceed 2,000 words or 4 published pages including figures, diagrams and references. The manuscript management system is completely online and includes a very quick and fair peer-review system, which is all easy to use. Visit http://www.dovepress.com/testimonials.php to read real quotes from published authors. 